\title{
A Framework Proposal for Blockchain-Based Scientific Publishing Using Shared Governance
}

\author{
Tim K. Mackey 1,2,3,4*, Neal Shah ${ }^{4}$, Ken Miyachi ${ }^{3,5,6}$, James Short ${ }^{3,7}$ and Kevin Clauson ${ }^{8}$ \\ ${ }^{1}$ Department of Anesthesiology, San Diego School of Medicine, University of California, San Diego, San Diego, CA, \\ United States, ${ }^{2}$ Global Health Policy Institute, San Diego, CA, United States, ${ }^{3}$ BlockLAB, San Diego Supercomputer Center, \\ San Diego, CA, United States, ${ }^{4}$ Department of Healthcare Research and Policy, UC San Diego - Extension, San Diego, CA, \\ United States, ${ }^{5}$ Institute of Electrical and Electronics Engineers, San Diego, CA, United States, ${ }^{6}$ LedgerSafe Corporation, \\ San Diego, CA, United States, ${ }^{7}$ San Diego Super Computer Center, San Diego, CA, United States, ${ }^{8}$ College of Pharmacy \\ and Health Sciences, Lipscomb University, Nashville, TN, United States
}

OPEN ACCESS

Edited by:

Sean T. Manion,

Science Distributed, United States

Reviewed by:

Hyojung Sun

Ulster University, United Kingdom

Wolfgang Lohmann,

PENTASYS AG, Germany

${ }^{*}$ Correspondence:

Tim K. Mackey

tmackey@ucsd.edu

Specialty section:

This article was submitted to

Blockchain for Good,

a section of the journal

Frontiers in Blockchain

Received: 20 July 2019 Accepted: 30 October 2019 Published: 15 November 2019

Citation:

Mackey TK, Shah N, Miyachi K

Short J and Clauson K (2019) A

Framework Proposal for

Blockchain-Based Scientific

Publishing Using Shared Governance.

Front. Blockchain 2:19.

doi: 10.3389/fbloc.2019.00019
Scientific research activity is reaching a staggering growth rate, introducing new and compounding existing challenges regarding the quality of peer-review, rise of predatory journals, and larger issues involving academic integrity and fraud stemming from the increased pressure to publish. Blockchain, a distributed ledger technology, is well-suited to address some of the challenges specific to scientific publishing. Companies including ARTiFACTS, Pluto, Orvium, and ScienceMatters-EUREKA, along with academic researchers, are exploring blockchain-based solutions to facilitate research data provenance and workflows, optimize the peer-review process, introduce better incentives, and even create new research journals and platforms utilizing blockchain. Building upon a review of these efforts, we propose a governance framework for scientific publishing based on a consortium blockchain model to create a more efficient means of navigating the publishing process. At the center of this framework is a model that adopts shared governance and validated inclusion via a Democratic Autonomous Organization (DAO). A DAO is an entity wherein the organizational rules are implemented and executed via smart contracts. The DAO will be comprised of participants of validated individuals and organizations who are publishers, editors, peer-reviewers, and citizen scientists to manage and oversee the framework. The framework also maps specifically to the publication workflow of submitting, handling, peer-review, and final editorial decision-making for scientific manuscripts. The goal of this framework is to increase transparency of scientific publishing, create a "pedigree" of a manuscript's research life cycle, and democratize the publication process while maintaining the accepted workflow common to scientific publishing by journals.

Keywords: blockchain, distributed ledger technology, academic publishing, scientific research, data governance, scientific integrity

\section{INTRODUCTION}

Each year there is an estimated $8-9 \%$ growth rate in research output driven by an increasing number of scientists and a concomitant rise in the pressure to publish with new journals being constantly launched to meet this demand (Noorden, 2014). Attempts to quantify research proliferation reveals staggering growth; data suggests that scientific 
output globally has doubled every 9 years since World War II (Bornmann and Mutz, 2015). In fact, the number of papers published hit a high of 2 million worldwide in 2015, with the largest growth in emerging markets including Brazil, India, China, and South Korea (AJE Scholarly Publishing Report, 2016).

Growth of research output is primarily driven by the hyper competitive environment of science (i.e., the idiom "publish or perish"), which has not only led to an increase in the number and variety of available publishing venues, but has also resulted in new business models for publishing (Rawat and Meena, 2014; Shamseer et al., 2017). One of the most fundamental changes is publishers moving from subscription-based to open access (OA) models that allow authors to retain rights to their works and makes them freely accessible to the public, but also imposes article processing fees (APFs) on authors (Solomon and Björk, 2011). Virtually all major publishers have OA options, including Reed-Elsevier, SpringerNature, and Taylor \& Francis, as well as top-tier journals published by the American Association for the Advancement of Science, the Journal of the American Medical Association, and the Lancet.

Despite increases in the number and options to conduct scholarly publishing, concerns regarding peer-review quality, plagiarism, absence of community and patient engagement, publication bias, predatory publishing, the cost of OA publishing, and the opaqueness of the "pedigree" of scientific research are ongoing challenges (Rawat and Meena, 2014; Shamseer et al., 2017). Broader issues of academic integrity, reproducibility, and preventing data falsification and fraud are also at the forefront of debate focused on ensuring that public trust is maintained in the scientific process (Nurunnabi and Hossain, 2019). Many of these issues originate from macro issues associated with the academy and scientific research, including power relations among researchers, pressure to publish for academic job placement and career advancement, and the potential influence of conflicts of interest in the publication process (Young, 2009; Marcovitch et al., 2010).

One technology that has the potential to address some but not all of these challenges is blockchain, a distributed ledger technology (DLT) with use cases across several industries (e.g., financial services, healthcare, supply chain, energy, education, etc.) (Mengelkamp et al., 2017; Treleaven et al., 2017; Chen et al., 2018; Kim and Laskowski, 2018; Mackey et al., 2019). At its core, blockchain is a decentralized, peer-to-peer network that creates an immutable, chronological record of assets with transactions that can be on a public, private, or consortiumbased blockchain (Mackey et al., 2019). Each "block" of data contains a "hash," which is a unique identifier of the block that can link to the hash of the preceding block-thus creating a cryptographically linked chain of data (Zheng et al., 2017; Mackey et al., 2019). This "block" of "chained" data can be distributed across blockchain nodes or participants and is subject to "consensus" of the network. A blockchain solution can also have many feature layers that operate processes on the blockchain network by interacting with other data architecture, including cryptocurrencies and tokens, distributed applications (DApps), and smart contract.
In this perspective, we explore existing efforts to use blockchain technology to improve scientific research with a focus on augmenting the current publishing processes. Based on a review of existing case studies and published research, we then formulate a new blockchain governance framework focused on increasing transparency in scientific publishing, creating a "pedigree" of a manuscript's research life cycle, and democratizing the publication process while maintaining the accepted workflow common to scientific publishing and journals.

\section{A REVIEW OF EXISTING COMMERCIAL AND RESEARCH APPROACHES}

In order to better understand the current and future landscape for blockchain-based publishing approaches, we examined both the gray literature for case studies of companies using blockchain for scientific research and conducted a nonsystematic review of multidisciplinary literature sources for life science/biomedical/health, engineering, and computer science articles for blockchain-related articles (see Table $\mathbf{1}$ for summary of case studies reviewed). We begin with a few case studies of companies that are attempting to use blockchain technology to address scientific research and the scholarly publishing process.

One goal of a scientific research blockchains is establishing provenance of research outputs, including tracking how these assets change through the research lifecycle. The blockchain startup company ARTiFACTS is developing a blockchain solution that records immutable chains of "scholarly" artifacts (e.g., figures, images, etc.) (ARTiFACTS., 2018). The purpose is to establish attribution and proof-of-existence of early scientific work, enable digital capture of research outputs, encourage sharing of data, and increasing impact of research content as it is created. This approach focuses on blockchain as an underlining technology to support, wherein research asset creation is timestamped and cryptographically hashed to improve the integrity of research content. Importantly, this approach is aligned with the "open science" movement, a concept central to OA publishing and which has support from numerous organizations and governments (United Nations Educational, Scientific and Cultural Organization, 2017). The ARTiFACTS system enables interfacing with existing open research data repositories such as GitHub and Figshare to better enable provenance, attribution, and trustworthiness of data. In this sense, ARTiFACTS focuses on one component of the scientific process; research asset creation and tracking and sharing. However, ARTiFACTS does not directly address specific challenges of the scientific publishing process. Similarly, the open source software Manubot enables blockchain-based manuscript version control. Version control is a critical component of the manuscript drafting and editing workflow but is also only one component of the scientific publishing process. There is an increased friction in the adoptability of non-comprehensive systems such as ARTiFACTS and Manubot.

Another company, Scienceroot, seeks to create a scientific blockchain-enabled ecosystem utilizing its own token [called "Science Token" (ST)], a digital wallet, and smart contracts 


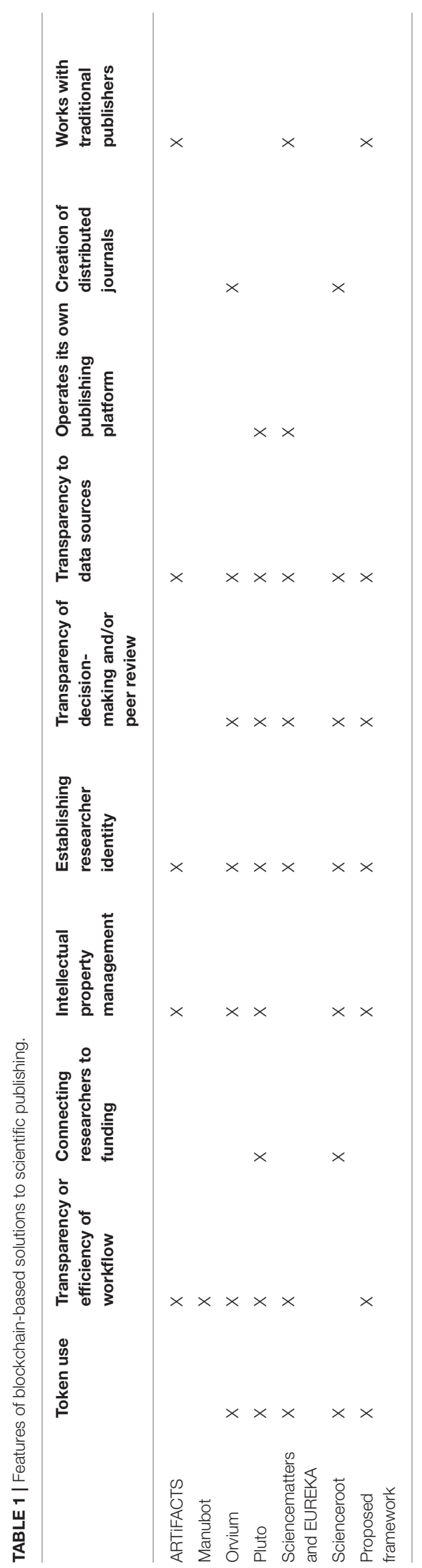

operating on a proof-of-stake consensus (Günther and Chirita, 2018). Research activities covered by the system include grant funding, publishing and scientific collaboration, with activities being linked to a researcher's identity through ORCID (the Open Researcher and Contributor ID), which acts as common digital identifier and is currently used for many research applications (Martin, 2018). Principally, Scienceroot describes itself as a decentralized collaboration platform, marketplace, and repository, while also proposing a decentralized open access scientific journal called "Scienceroot Journal." Scienceroot's proposed solution is broad, including a publishing model where it runs its own journal(s) and requires APFs in the form of tokens. The difference with existing open access journals is that Scienceroot states it will use token revenue and a reward sharing program to redistribute APFs STs to reviewers and authors while also encouraging ST donations (Günther and Chirita, 2018). Scienceroot's system is interesting, though heavily relies on tokenization to drive the research process. Another possible limitation is the decision to use its own blockchain branded journal as a publishing platform instead of using existing publishers that are already established, are indexed, and may already have an impact factor.

Similarly, the blockchain company Pluto proposes a decentralized ecosystem to manage transfers of value through the research lifecycle by using smart contracts and tokens on the blockchain and smart contract environment Ethereum. Pluto's system allows users to submit and store different types of scientific information/data (with Digital Object Identifiers) and retain copyright control. It also includes a "reputation score" to quantify user research contributions and a "blinded" and open peer review process for deposited scholarly submissions (Pluto Network, 2018). Research is subject to peer review with "reward" tokens and reputation provided in substitution for APFs that are distributed to reviewers. Different from Scienceroot, Pluto appears to decentralize the scientific communication process by decoupling from a traditional scientific "journal" approach and instead disseminating content directly on its online platform. In this sense, Pluto may not fit the narrow definition of scientific publication through a journal article format. This fundamental difference may limit adoption given that scientists continue to focus on publication in journals, conference proceedings, books, and other publication medium that are familiar to the research community and are recognized in academic promotion and professional development.

The blockchain-based publishing platform Orvium differs from other models by focusing on integrating blockchain technology into the publication life cycle while also encouraging open-science and research dissemination aims. The platform states its software will be developed open source with the lifecycle processes driven by a Orvium token as a reward system (Orvium, 2019b). Key features of the proposed system include immediate proof-of-existence upon submission, transparent and open peerreview, and the ability to transfer copyrights and licenses by authors (Orvium, 2019b). The platform aims to address several publishing challenges, including lowering the cost of publication and access, creating better rewards and incentives for peer reviewers, improving transparency in the peer review process, 
and enabling better sharing of research data (Orvium, 2019b). Platform components described in the minimally viable product (MVP) for the platform include frontend applications (i.e., web applications), backend applications (e.g., APIs), the blockchain environment itself, and use of databases for storage (Orvium, 2019a). Unique to this approach is the ability for individuals and institutions to create Decentralized Autonomous Journals (DAJs) with their own governance rules and licensing and subscription models. In this sense, Orvium provides a platform for creation of new DAJs, but it is not clear if it's blockchain environment will also integrate with existing publishers or journals. Hence, adoption of Orvium may focus on the success and uptake of DAJs as a new journal entity type among the scientific community.

Another company, ScienceMatters, operates an open-access publishing platform that focuses on single observation studies (and also encourages negative and replication studies). The publishing platform currently operates two OA journals and also uses Ethereum to operate its companion EUREKA solution (see more below), a scientific review and rating platform that uses tokens (ScienceMatters, 2018). ScienceMatters offers an interesting approach, as it uses a blinded blockchain peer-review process with authors paying an upfront fee of $\$ 595$ to pay for the cost of reviewers who are then paid with Eureka tokens (with partial refund if rejected.) This allows it to operate a web-based OA publishing platform that works in tandem with a journal submission and token reward system powered by blockchain. Submissions are also timestamped, peer-review is crowdsourced, scientific work evaluated and assigned a metric, and artificial intelligence (AI) approaches will be used for research matching purposes.

The ScienceMatters and EUREKA platform is arguably one of the most robust blockchain-based publication solutions described, as it operates an existing OA publishing web frontend and uses blockchain as a means to adjudicate the peer-review and journal submission process. However, ScienceMatters primarily promotes the use of its own open access journal platform, not established publishers, though there is an option to transfer immutable rating and evaluation scores to other journal outlets. It is unclear whether these blockchain features will enable the ScienceMatters journal platform to attract submissions, whether its focus on single observation studies will address incentives for researchers to publish these types of articles, whether there will be enough submissions to power AI-based research matching, or whether other journals or publishers will accept EUREKA ratings or incorporate them into their own submission and review decisions.

\section{Published Literature}

In addition to commercial and startup activity, there are a handful of research articles exploring blockchain's application to journal article submissions. A non-systematic literature review of PubMed (medical and life science journals), IEEE Xplore (engineering journals), and the ACM digital library (computer science articles) using keywords including "blockchain," "education," "research," and "publication" found that most of the literature on blockchain technology focuses on use cases involving data sharing and privacy, integration with Internet of Things (including mobile), issues related to cryptocurrencies and tokens, technical issues (e.g., consensus mechanisms and permission structures), business process management, interaction of blockchains with artificial intelligence, data storage and security, and blockchain use cases in industry verticals such as supply chain, energy, transportation, and healthcare. There are far fewer articles that focus on education or research management, and there were only three papers we found that were specific to the journal publication process (Bore et al., 2017; Duan et al., 2017; Hoy, 2017; Skiba, 2017; Bai et al., 2018; Han et al., 2018; Kleinaki et al., 2018; Turkanović et al., 2018; Agbo et al., 2019; Al Harthy et al., 2019; Conard, 2019; Verde et al., 2019).

The three relevant papers reviewed were conference papers and were retrieved from IEEE Xplore. The first article coauthored by Gipp et al. describes "CryptSubmit," a bitcoin blockchain system to create a publicly accessible timestamp of manuscript submissions integrated into the open source conference management system Open Journal System (OJS) (Gipp et al., 2017). CryptSubmit was also designed to enhance intellectual property protection of authors via traceability of ideas/knowledge and to improve access to electronic pre-print and storage services (Gipp et al., 2017). This system is similar to other solutions that address only one component of the publication process and its related challenges.

A second and third paper by Niya et al. and Schaufelbuhl et al. describes the aforementioned EUREKA platform in more technical detail and provides an explanation of its broader potential utilization. Importantly, it first identifies primary scientific publishing challenges that the system seeks to address including publishing negative/null result studies, lack of credit for scientific contributions, and issues of reproducibility/reusability (Niya et al., 2019; Schaufelbühl et al., 2019). EUREKA is based on a public blockchain design on Ethereum operationalized with a front-end interface, smart contracts, DApps, and tokens to incentivize participation. It maps its operation to six steps of the journal submission process, while also introducing other features such as crowd-sourcing reviewers and matching reviewers based on AI and text mining as previously described (Niya et al., 2019). Limitations of the system are similar to those outlined above.

Finally, a 2018 Nature Index editorial sums up the general use cases for blockchain in scientific publishing that are being collectively explored by commercial activities and as described in published research. These use cases broadly focus on: (1) transparency of journal management workflows; (2) storing and curating data; (3) connecting researchers to funding opportunities; (4) managing intellectual property; (5) establishing identity and preventing fraud (including need for reproducibility); (6) transparency in scientific decision making; and (7) transparency to data sources and methods behind journal metrics (Brock, 2018) Early commercial and pre-commercial work combined with a growing body of blockchain research indicates that blockchain could be a potentially disruptive technology in scientific publishing. Despite this potential, scholarly publishing is still driven by many conventions and norms that are difficult to change. 
Hence, it remains unclear if these blockchain approaches will be "fit-for-purpose" for the specific needs of academic institutions, researchers, publishers and long-standing traditions that continue to persist.

\section{A PROPOSED SHARED GOVERNANCE FRAMEWORK FOR BLOCKCHAIN SCIENTIFIC PUBLISHING}

\section{Aims of Framework}

Building on existing case studies and published research, we propose a governance framework for blockchain-based scientific publishing that we will pilot in a future research project led by co-author investigators at the San Diego Supercomputer Center BlockLAB. Our governance framework builds on earlier approaches, such as Orvium and EUREKA, but differs from these examples by focusing on a architectural framework that integrates and augments processes performed by traditional publishers and scientific journals in lieu of creating a new blockchain-based publishing platform or journal entity (i.e., DAJs). The goal of the framework is to enhance the transparency and integrity of publishing through shared governance between publishers, scientists, and the public by mapping the publication workflow to blockchain architecture, while being agnostic to a specific publisher (see Figure 1).

We choose blockchain over other technologies-such as cloud computing and solutions relying on traditional data storage mechanisms-because our framework requires data provenance of journal submission history, process-based workflow transparency, validation of participating users, and establishing rules-based consensus among participating actors in a decentralized network. Though other technologies can enable data sharing through permission structures, create process workflows, and support distributed networks, the ability of blockchain to leverage cryptography, DApps, smart contracts, and interact, store and publish data via on-chain and off-chain approaches, positions it as a unique combination of technology tools for specific challenges we seek to address in scholarly publishing.

The scientific publishing challenges that this framework aims to address are: (1) enabling better detection of issues related to unethical and potentially fraudulent practices in order to improve the integrity of the publication process; (2) creating a more efficient and equitable publication and peer-review process (including better sharing of resources, and enhancing quality and speed in peer-review); (3) encouraging broader engagement in research for the public and citizen scientists; and (4) creating an environment enabling shared governance where communities reach consensus on the appropriate rules and norms of the academic publication process.

The framework addresses these challenges respectively by: (a) improving transparency and oversight of the submission, adjudication and publication history for manuscripts agnostic to specific journals/publishers; (b) mapping publication workflows to smart contracts and token payments; (c) inclusion of a community of citizen scientist in the shared governance and as peer-reviewers; and (d) creating a DAO to enable decentralized and shared governance driven by validated nodes of users (see more below). Some of these features could also potentially address issues related to lack of reproducibility, conflicts of interest in publishing, and the need for confirmatory or negative/null result studies but are not the main focus of our framework. Other macro issues, such as power dynamics in research/authorship and the pressure to publish, are also not directly addressed.

\section{Design Elements}

Importantly our proposed framework departs from other proposals as it does not decouple the governance process from traditional publishers and journals. Other blockchainbased publication solutions discussed primarily focus on creating their own OA journals or research platforms that interact with blockchain systems or new blockchain-powered journals. Based on our focus, we will use a consortium blockchain design (including elements of both a private permissioned blockchain and a public blockchain) that can include participation from existing journals and publishers that contribute together in a shared ecosystem with authors, reviewers, and editors under shared governance principles.

The primary design components of the governance framework is based on an integration approach with existing publishers. The framework will consist of a submission web frontend layer, the Ethereum network which executes the smart contract and blockchain storage aspects of the framework, and the backend database that stores off-chain interactions and data (see Figure 2 for design summary). For the first layer, we will focus on developing both data and UI/UX integrations with existing journal submission portals (e.g., ScholarOne, Editorial Manager, and OJS) through use of APIs that can transmit individual submission data in JSON format off-chain. For example, user login and identity verification will leverage ORCID integration via ORCID registry and member API credentials. This integrated frontend layer will communicate directly with the framework's Ethereum Network governed by a Democratic Autonomous Organization (DAO). A DAO is an entity wherein the organizational rules are implemented and executed via smart contracts. Submission metadata can also be submitted directly to the framework via a dedicated generic frontend web application that connects directly to the Ethereum Network and backend database. In this case, the submission will be journal agnostic and journal selection can occur later.

The backend database will house off-chain data (including submission data generated from the frontend and publication workflow data not published on-chain), will act as the primary analytical engine for data analysis, and will also interact with the Ethereum network. Permission structures governed by public and private keys will dictate access to non-public on-chain and off-chain data based upon agreed roles of participants and any assigned validating nodes during different phases of the publication workflow. For consensus the Ethereum network will utilize a proof-of-authority (POA) consensus mechanism. POA, a modified version of Proof of Stake, uses the validator's identity as a form of stake to validate blocks. In this sense, consensus 

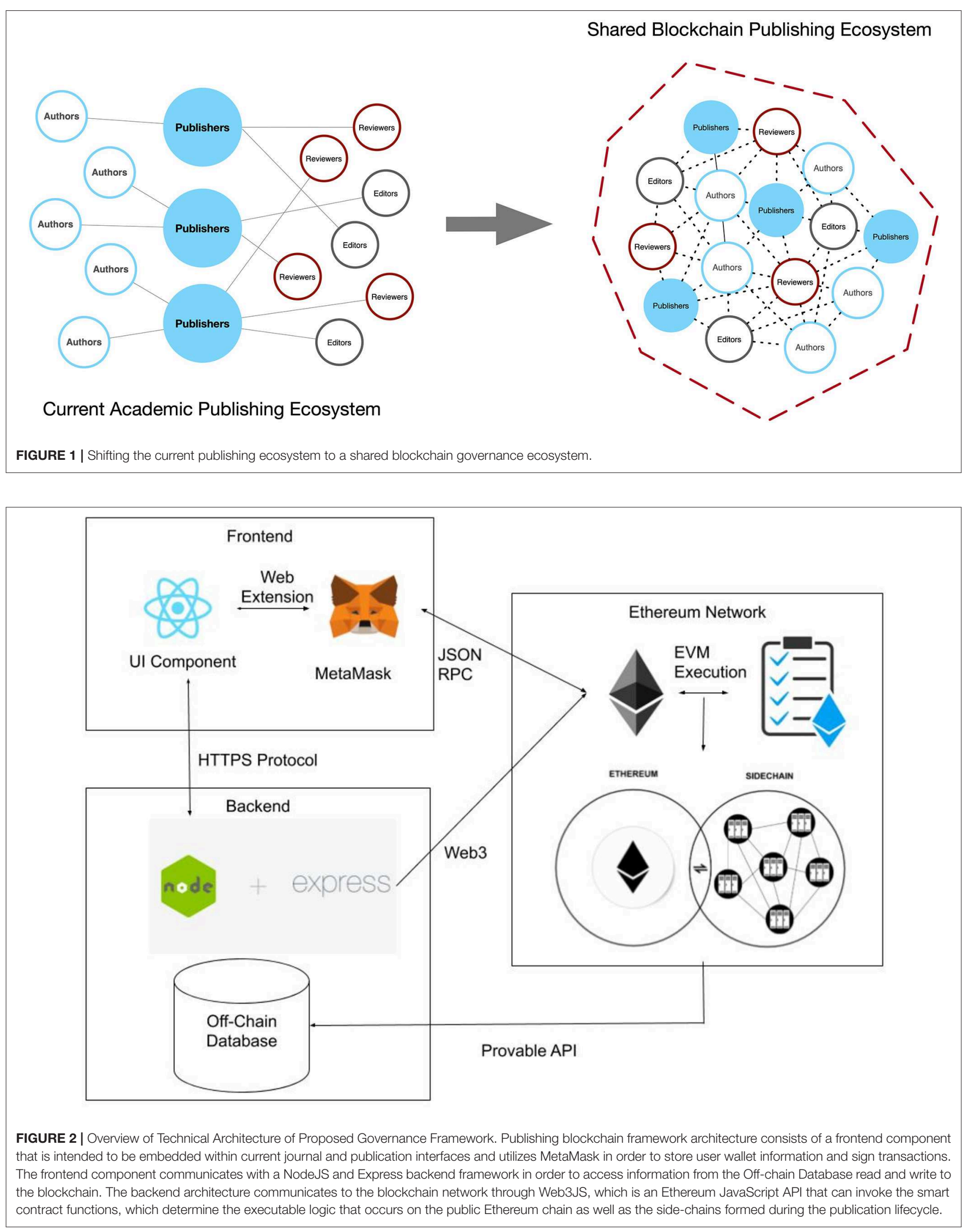


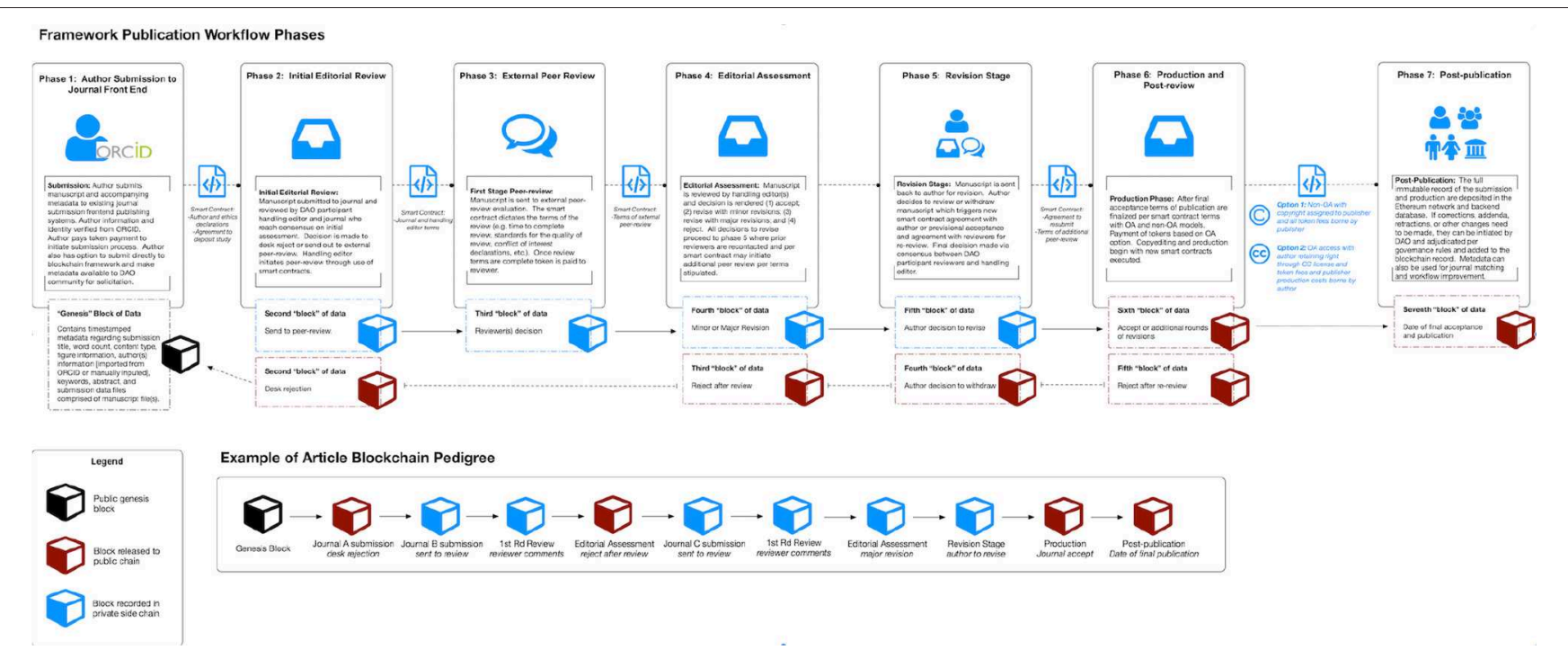

FIGURE 3 | Overview of Publication Workflow of the Framework. Publishing blockchain workflow overview. This process starts with the creation of a "genesis" block and subsequent blocks of data that are "chained" together to form the complete submission history pedigree for a manuscript.

of decisions on the framework (e.g., editorial decisions, peerreview, and final acceptance/rejection of manuscripts) will be public and tied to a user's identity with decisions made based on DAO-agreed upon voting rules (e.g., majority of nodes or weighted voting) for different workflow decision points. Candidates for POA validation nodes will need to be participants with validated identity (e.g., ORCID) in the DAO and may have their validation privileges revoked in cases of unethical behavior that can be more easily detected due to the transparent nature of public data published on-chain and visibility to consensus.

The Ethereum Network will also include two key application layers; a platform-specific ERC-20 token for quantification and payment of publication workflow activities; and a suite of smart contracts to execute publication-specific management workflows (e.g., ICMJE author disclosures, copyright/CC, etc.) as described in the next section. Shared governance of the platform is conducted via the DAO comprised of separate groups of validated publishers, editorial board members, handling editors, reviewers, and the public. Membership for individual authors/reviewers/editors/citizen scientists and institutional publishers in the DAO is validated by peers in the decentralized community based on expertise and credentials (e.g., ORCID research profile data, Publons.com for review history, institutional and educational background). DAO participants can also be further sub-grouped into different scientific discipline or expertise areas. These DAO participants will be the "Authority" nodes that will validate the scientific publishing information that is proposed to be written to the blockchain. This validation of both DAO participants and authority nodes will better ensure that fraudulent journals (e.g., predatory journals) and/or inexperienced or reviewers with questionable or fraudulent review history do not participate in the shared governance framework.

\section{Academic Publication Workflow}

Importantly, our proposed framework and its related architecture maps to the publication workflow of submission, review, acceptance, and dissemination in seven phases. Each phase represents a new transaction event that creates a timestamped cryptographically hashed "block" of data as outlined below (see Figure 3 for summary).

\section{Phase 1: Author Submission}

The first phase consists of manuscript submission to the existing journal frontend through an ORCID Member webfront publishing system. All submissions are created with a genesis block upon the first submission, which is then linked to subsequent submissions if the manuscript metadata matches. The author (identity validated by ORCID) submits the manuscript and submission metadata via the normal journal submission process with the data integration framework API transmitting the ORCID author identity and submission metadata to the Ethereum Network and the backend framework database. Authors can also submit the submission metadata directly to the Ethereum network via a generic web frontend where the submission is not assigned to a journal but instead to a specific DAO participant community who can independently evaluate submissions for fit and rigor. This would be similar to an open peer-review format prior to publication with evaluation open to an entire DAO participant discipline specific community. Editors or publishers in turn, may determine that a solicitation or invitation to submit is appropriate through this process.

The system will also use submission metadata stored offchain (including title of article, keywords, text of abstract, content type of manuscript, and word count) to suggest potential matching journals that are appropriate to the manuscript subject matter, length and format. This can be accomplished using 
combined approaches in data analytics, topic models, natural language processing, and machine learning that are carried out on the backend framework database. Similar approaches are used by the website JANE (available at: http://jane.biosemantics. org) developed by the Biosemantics Group, which calculates a similarity and confidence score to match title or abstract text of a manuscript to possible PubMed journals. Feature engineering to match a submission topic area, title, content type, prior publications in a journal, and other features can be explored.

Formal submission to a journal will follow a single submission protocol with select submission metadata (author name and contact information, date of submission, title, submitted journal name) written to the public blockchain that is timestamped immediately upon verification, while other potentially confidential information will reside offchain. A private side-chain blockchain gets instantiated wherein the initial information is deposited into a genesis block. The private side-chain is intended to track the lifecycle and provenance of the submission without publicly releasing all of the information. A smart contract executes required author declarations and agreement to deposit the submission in the system for a nominal token submission fee paid by submitted author. Importantly, the terms of the submission, including ownership of copyright, token costs of publication workflow, and accessibility, are transparently disclosed and agreed upon by author, publisher, and handling editor.

\section{Phase 2: Manuscript Handling}

In Phase 2, after the article has been submitted to a journal, the subsequent adjudication history will remain in a private side-chain through different phases of the editorial and peer-review process. We propose that DAO participants of journals/publishers, handling editors, and reviewers collectively determine token costs for manuscript handling that can also be journal or publisher specific based on the specific terms of the publication process. This token cost should cover the direct and reasonable fees for peer-reviewers and handling editors covered by token submission fees from authors and also by publishers depending on terms of publication (explained later).

Following deposit, the submission is handled by the journal's internal editorial staff or a pool of validated independent handling editors with at least two validating nodes (a publisher and handling editor) reaching consensus on deciding whether a submission is sent out for external review or rejected. This creates a second block of data with the linked hash of the genesis block and a pointer to metadata recording the "desk" decision and identity of POA validators. If rejected, this block is released to the public chain permanently recording it as part of the manuscript's transaction history (i.e., an article submission with a desk edit rejection by Journal $\mathrm{X}$ and handling editor Y). If the article is accepted for external review, then a smart contract is executed between the journal and handling editor recording the type (single or double blinded, or open review) and editorial responsibilities for review and the terms of token compensation.

\section{Phase 3: External Peer-review}

The third phase begins the process of external peer-review. The handling editor selects from a pool of DAO external peer-reviewers who are matched based on their research metadata and ORCID profiles or can be invited by handling editors if they are DAO validated members or can be subsequently validated following invitation. In addition to scientific reviewers, an optional citizen scientist can be chosen to assess implications of research from a public or patientcentric perspective. For example, the medical journal BMJ operates the BMJ's Partnering with Patients initiative, which helps to include patient editors and reviewers in the publication process. A smart contract is executed upon external reviewer acceptance also detailing review terms based on publisher requirements (such as time to complete, structure of review, and conflict of interest declarations). Once review is complete and terms are met, tokens are deposited as payment to external reviewers. After external review, a third "block" is created with pointers to reviewer identity, decision of review, and a pointers to the content of the review (comments to author and editor) data.

\section{Phase 4: Editorial Assessment}

The fourth phase is editorial assessment following external peer review. Decisions and recommendations made by the external reviewers are assessed by handling editor with a decision to accept, revise with minor or major revisions, or reject the manuscript on the basis of external review. Consensus is established by POA with the majority of external reviewer and handling editor nodes reaching agreement. This would ensure handling editor decisions are transparent and made in congruence with external reviewer assessments. If the manuscript is rejected, phase 2-4 blocks are released to the public chain and the submission is released. Importantly, if a manuscript is rejected or subsequently withdrawn, the author pays the token cost for external review and the publisher pays the prorated token costs for handling editor's work up to that phase. This fourth block includes pointers to data recording the editorial decision, editorial decision-making comments, and deadlines for revisions by authors. A smart contract is executed to secure external reviewers for re-review if necessary.

\section{Phase 5: Revision Stage}

The fifth phase is the revision and re-review stage. The author will either revise and resubmit the manuscript or withdraw the manuscript from consideration. If the author chooses to withdraw, then transaction data is released to the public chain. If the author elects to revise and resubmit, they will deposit a revised article (with pointers to document for blockchain versioning control) and a point-by-point response rebuttal. The manuscript is then sent out to handling editor and external reviewers for re-review to reach consensus on a decision. Phase 3-5 processes are 
repeated until a final decision is made, where a block of data (simplified for illustrative purposes as the fifth block) is created with pointers for data on the decision to accept for publication, proof of consensus by handling editor and external reviewers, and publisher's general terms for publication acceptance.

\section{Phase 6: Production and Post Peer-Review}

The sixth phase marks initiation of the production phase. A smart contract executes the terms agreed upon and disclosed in phase 1 between author and publisher or via a subsequent publication agreement between author and publisher when directly making a submission to the framework. If the author and publisher have agreed to non-OA (with copyright assigned to publisher), the submission token fee and all token fees for handling editor/external reviewers are automatically assigned to the publisher (including any retrospective payments to author). If author elects for OA (author retains copyright/Creative Commons license) then author pays the direct token cost for handling editor, reviewers, and any production costs previously disclosed by publisher upon submission in the form of a combined token-based APF. The sixth "block" of data contains a pointer to the agreement and terms of publication, records the token transactions to all parties, and provides the final data/time of acceptance. After acceptance, all blocks related to the adjudication history for the accepting journal are released to the public chain on the Ethereum network with a duplicate copy published to the backend database.

\section{Post Publication Phase}

In the post-publication phase, the immutable blockchain hash chain of the manuscript's complete adjudication is released, which can include multiple submissions and decisions. This includes the hash chain associated with a single submission and all submissions associated with the genesis block that were re-released to the public chain (i.e., previous rejections of a submission). Information on the blockchain is now public and includes the entire history related to identity (depending on terms of review), comments, and decisions of editors and reviewers, and proof of consensus via POA in adjudication. Publishers are encouraged to deposit preprints of accepted manuscripts generated in Phase 6 to both the DAO and to the general public. Journals and their publishers then move forward with publication of accepted articles on their own publishing platforms with pointers or hyperlinks to the public on-chain transaction log so the public can review the published article's full submission and adjudication history. The blockchain data of the article's pedigree should also be linked to the indexed record on scientific repositories and databases (e.g., PubMed, IEEE Xplore, JSTOR, ACM Digital Library, SCOPUS, etc.) If retractions or corrections are needed, consensus of publishers and handling editors can be sought post-publication based on history available, and new information appended to the published blockchain record.

\section{DISCUSSION}

In developing our shared governance framework for blockchainbased scientific publishing we focused on three core principles aimed at enhancing transparency, accountability, and trust in academic publishing by: (1) retaining the traditional model of scientific publishing via journals while leveraging blockchain technology features to improve the efficiencies of the publication workflow; (2) establishing an immutable and fully transparent history of published and unpublished research; and (3) fostering a decentralized community focused on shared governance with validated nodes of authors, reviewers, editors and publishers in the DAO. Our framework aims to create a publisher-agnostic ecosystem allowing groups of validated participant stakeholders to eventual self-govern and agree on rules and norms of how to equitably participate in a decentralized scientific publishing community.

Inclusiveness based on consensus and validation is a key theme of this framework. The governance approach using DAO participants for respective stakeholder communities of validated researchers, publishers, editors, reviewers, and citizen scientists enables shared accountability. Under this system, any publisher validated by the scientific community, including large group to mid-size, and smaller professional societies who operate independent journals, could participate as a publisher node. It also opens the door to validated handling editors who are independent of journals, professionalization of reviewers who are dedicated and produce high quality reviews for token payments, and citizen scientists who can add important perspectives and meaningful contributions to incorporate into research.

Validation of DAO participants also ensures that bad actors or predatory journals will have a harder time participating, and if questionable or unethical conduct occurs, nodes can be removed or lose their validating privileges under POA which is identity-based. The DAO can also operate autonomously from established publishers and reach shared consensus on establishing governance rules and norms around the function, ethics, and operating procedures of the framework making it more democratized. For example, setting the token cost for article submissions or external reviewers is a decision that should be made by the DAO based on what is deemed equitable and the actual cost and time of processes, not simply on a publisher's own profit margin. This shared accountability could also create transparency around the actual cost of publishing and ensure delinkage of considerations of $\mathrm{OA}$ revenues from editorial decision making.

Another key benefit of our framework is enhanced transparency through establishing article history provenance by publishing blocks of data for the adjudication history of submissions after decisions are rendered. Upon rejection or acceptance, blockchain transactional data becomes public, enabling availability of the current history of the manuscript's submission, rejection, and acceptance (including timestamped data and identity of journals). This can aid 
in detection and reporting cases of research fraud or impropriety (such as multiple or concurrent submissions, detection of plagiarism, unethical use of reviewers or soliciting fake reviewers, repeat submission of same result studies, concerns expressed by reviewers regarding data integrity, etc.) and other red flags that can be detected by the DAO possibly preventing fraudulent studies from being published. Currently this data is not published publicly or linked, with journal submission history largely siloed in single journal submission systems.

The framework also ensures a single immutable record for a manuscript's pedigree that can be appended in an article's post-production history for corrections, erratum or retractions if needed. Further, public metadata available from manuscript submission histories can help researchers better identify journals and reviewers knowledgeable about specific research areas, potentially accelerating scientific publication through improved targeting and matching of journals, handling editors, and peer reviewers.

\section{Possible Limitations and Challenges}

Importantly, we designed our framework to leverage the potential of blockchain technology but did not completely decouple traditional publishers from the workflow or shared governance model. We chose this design as scientists continue to make decisions about journal submission based on traditional journal parameters/characteristics, such as indexing, topic match, acceptance/rejection rate, and impact factor (Welch, 2012; Nagarajappa and Wahrekar, 2017). Though innovative approaches to academic publishing, peer-review, and measuring scientific impact are starting to emerge, scientists, as well as academic promotion and review committees, continue to prioritize publications from prestigious journals as a proxy for quality and rigor (Ravenscroft et al., 2017; Schimanski and Alperin, 2018). Hence, scientific blockchain approaches that create their own independent $\mathrm{OA}$ publication platforms integrated with blockchain solutions or new blockchain-powered journals (i.e., DAJs) will likely face challenges in attracting sufficient article submissions. This is a challenge for all new scholarly journals, but particularly problematic when a platform relies on active collaboration and token funding to drive publishing processes. Failure to attract authors could also impact the ability of a platform/journal to secure indexing and an impact factor.

However, continuing to rely on traditional publishing venues also has its limitations, risks, and associated challenges. Established publishers may not want to participate in our proposed framework. Instead, they might prefer to keep all editorial processes, decisions, and systems within their purview. There are good arguments for this approach as many publishers harmonize submission and review processes across their families of journals, and similarly train their editorial staff based on specific publisher's policies and style guidelines to ensure consistency, quality, rigor and integrity. Publishers may also not see the value of transparency or more efficient processes when taking into considerations cost of new technology adoption, concerns about security, and the need for data integration with legacy systems. More importantly, academic publishing is a multibillion dollar industry, and it is unclear if private companies who operate their own publishing ecosystems for profit, would ever participate in systems that delegate some of their authority on issues such as publishing costs, paying reviewers, or outsourcing editorial decision making, even if these processes were more inclusive (Buranyi, 2017). In fact, these barriers may be primary drivers of why existing blockchain case studies reviewed are focusing on developing their own publication platforms independent of traditional journal models.

Additionally, though shared governance sounds ideal, in practice, reaching consensus on specific issues related to scientific publishing across a set of diverse actors will likely be challenging for the DAO. Publishers, authors, and reviewers represent very different stakeholder groups with different incentives for participation and rules and norms may also need to be discipline specific. Fundamental differences in opinion about the way scientific publishing should be conducted may impede the DAO from reaching consensus on key governance issues of the framework, such as which nodes validate, setting voting rules for POA during different phases of the publication workflow, and setting token costs and values. Interestingly, though our framework envisions an inclusive consortiumbased blockchain of the entire scientific community, it may be more pragmatic for the framework to be piloted as a private blockchain for a single publisher or journal family. This could simplify participation in the DAO to publisherassociated editors and reviewers and smart contract processes specific to a publisher, though this is not the focus of our approach.

Additionally, our framework is not entirely novel, as it adopts many of the same approaches as other blockchain scientific research and publishing case studies discussed. For example, our framework relies on tokens to drive transactions and user participation, so is subject to similar limitations of not fully understanding how this could impact real-world behavior as a result of conflicts-of-interest, negative externalities, inefficiencies due to gaming the system, and/or fail to properly incentivize positive behavior. The framework design is also limited to future submissions and as currently proposed would not incorporate retrospective submission history, though validation of historical submission data using blockchain approaches could be explored.

\section{CONCLUSION}

The framework we have developed builds upon an emerging body of innovation seeking to transform scientific publishing using blockchain technology. However, all of these proposals, including our own framework, face barriers to adoption and implementation that require further experimentation, assessment and active collaboration with the scientific community. Importantly, these challenges should not discourage continued distributed science advancements, as blockchain is a technology that has both disruptive and 
transformative potential to address issues core to research integrity and ensuring public trust in science. We believe that blockchain technology has the potential to revolutionize scientific publishing, as long as it focuses on the "three D's" of "distributed," “decentralized," and "democratized," research under a future shared governance framework.

\section{REFERENCES}

Agbo, C. C., Mahmoud, Q. H., and Eklund, J. M. (2019). Blockchain technology in healthcare: a systematic review. Healthcare 7:56. doi: 10.3390/healthcare7020056

AJE Scholarly Publishing Report (2016). A Brief Look at Current Publication Trends, Popular Areas of Study, and the Journals and Institutions With the Most Publications. Available online at: https://www.aje.com/dist/docs/ International-scholarly-publishing-report-2016.pdf

Al Harthy, K., Al Shuhaimi, F., and Al Ismaily, K. K. J. (2019). "The upcoming blockchain adoption in higher-education: requirements and process," in 2019 4th MEC International Conference on Big Data and Smart City (Muscat: ICBDSC).

ARTiFACTS. (2018). ARTiFACTS Launches First-Ever Blockchain-Based Platform for Scientific and Scholarly Research. PR Newswire. Available online at: https://www.prnewswire.com/news-releases/artifacts-launchesfirst-ever-blockchain-based-platform-for-scientific-and-scholarly-research300615989.html

Bai, Y., Li, Z., Wu, K., Yang, J., Liang, J., Ouy, B., et al. (2018). "Researchain: union blockchain based scientific research project management system," in 2018 Chinese Automation Congress (CAC) (Xi'an).

Bore, N., Karumba, S., Mutahi, J., Darnell, S. S., Wayua, C., and Weldemariam, K. (2017). "Towards blockchain enabled School Information Hub," in Proceedings of the Ninth International Conference on Information Communication Technologies and Development (Lahore).

Bornmann, L., and Mutz, R. (2015). Growth rates of modern science: a bibliometric analysis based on the number of publications and cited references. J. Assoc. Inf. Sci. Technol. 66, 2215-2222. doi: 10.1002/asi.23329

Brock, J. (2018). Could Blockchain Unblock Science? Available online at: https:// www.natureindex.com/news-blog/could-blockchain-unblock-science

Buranyi, S. (2017). Is the Staggeringly Profitable Business of Scientific Publishing Bad for Science? The Guardian. Available online at: https://www.theguardian. com/science/2017/jun/27/profitable-business-scientific-publishing-bad-forscience

Chen, G., Xu, B., Lu, M., and Chen, N.-S. (2018). Exploring blockchain technology and its potential applications for education. Smart Learn. Environ. 5:1. doi: 10.1186/s40561-017-0050-x

Conard, S. (2019). Best practices in digital health literacy. Int J Cardiol. 292, 277-279. doi: 10.1016/j.ijcard.2019.05.070

Duan, B., Zhong, Y., and Liu, D. (2017). "Education application of blockchain technology: learning outcome and meta-diploma," in 2017 IEEE 23rd International Conference on Parallel and Distributed Systems (Shenzen).

Gipp, B., Breitinger, C., Meuschke, N., and Beel, J. (2017). "CryptSubmit: introducing securely timestamped manuscript submission and peer review feedback using the blockchain," in 2017 ACM/IEEE Joint Conferences on Digital Libraries (Toronto, ON).

Günther, V., and Chirita, A. (2018). "Scienceroot" Whitepaper. Available online at: https://www.scienceroot.com/resources/whitepaper.pdf

Han, M., Li, Z., He, J., Wu, L., Xie, Y., and Baba, A. (2018). “A novel blockchainbased education records verification solution," in Proceedings of the 19th Annual SIG Conference on Information Technology Education (Fort Lauderdale, FL).

Hoy, M. B. (2017). An Introduction to the blockchain and its implications for libraries and medicine. Med. Ref. Serv. Q. 36, 273-279. doi: 10.1080/02763869.2017.1332261

Kim, H. M., and Laskowski, M. (2018). Toward an ontology-driven blockchain design for supply-chain provenance. Intell. Syst. Account. Finance Manage. 25, 18-27. doi: 10.1002/isaf.1424

Kleinaki, A. S., Mytis-Gkometh, P., Drosatos, G. L., Efraimidis, P. S., and Kaldoudi, E. (2018). A blockchain-based notarization service for

\section{AUTHOR CONTRIBUTIONS}

TM, KM, and NS jointly collected the data, designed the study, conducted the data analyses, and wrote the manuscript. TM, NS, KM, JS, and KC contributed to the formulation, drafting, completion, and approval of the final manuscript.

biomedical knowledge retrieval. Comput. Struct. Biotechnol. J. 16, 288-297. doi: 10.1016/j.csbj.2018.08.002

Mackey, T. K., Kuo, T.-T., Gummadi, B., Clauson, K. A., Church, G., Grishin, D., et al. (2019). "Fit-for-purpose?" - challenges and opportunities for applications of blockchain technology in the future of healthcare. BMC Med, 17:68. doi: 10.1186/s12916-019-1296-7

Marcovitch, H., Barbour, V., Borrell, C., Bosch, F., Fernández, E., Maconald, H., et al. (2010). Conflict of interest in science communication: more than a financial issue. Croat. Med. J. 51, 7-15. doi: 10.3325/cmj.2010.51.7

Martin, M. (2018). Reinvent Scientific Publishing With Blockchain Technology. Available online at: https://www.statnews.com/2018/12/21/reinvent-scientificpublishing-blockchain/

Mengelkamp, E., Notheisen, B., Beer, C., Dauer, D., and Weinhardt, C. (2017). A blockchain-based smart grid: towards sustainable local energy markets. CSRD. 33, 207-214. doi: 10.1007/s00450-017-0360-9

Nagarajappa, S., and Wahrekar, S. (2017). Choosing the scientific journal for publishing research work: perceptions of medical and dental researchers. Clujul. Med. 90, 196-202. doi: 10.15386/cjmed-704

Niya, S. R., Pelloni, L., Wullschleger, S., Schaufelbühl, A., Bocek, T., Rajendran, L., et al. (2019). "A blockchain-based scientific publishing platform," in 2019 IEEE Conference on Blockchain and Cryptocurrency (ICBC) (Seoul).

Noorden, R. V. (2014). Global Scientific Output Doubles Every Nine Years. Nature Newsblog. Available online at: http://blogs.nature.com/news/2014/05/globalscientific-output-doubles-every-nine-years.html

Nurunnabi, M., and Hossain, M. A. (2019). Data falsification and question on academic integrity. Account. Res. 26, 108-122. doi: 10.1080/08989621.2018.1564664

Orvium (2019b). Whitepaper: Accelerated Scientific Publishing (v1.7). Available online at: https://docs.orvium.io/Orvium-WP.pdf

Orvium (2019a). Architecture \& Technology Specification: Accelerated Scientific Publishing v(1.2). Available online at: https://docs.orvium.io/Orvium-PA.pdf

Pluto Network (2018). White Paper Draft (ver 0.4). Available online at: https:// assets.pluto.network/Pluto_white_paper_v04_180719_1355_BSH.pdf

Ravenscroft, J., Liakata, M., Clare, A., and Duma, D. (2017). Measuring scientific impact beyond academia: an assessment of existing impact metrics and proposed improvements. PLoS ONE 12:e0173152. doi: 10.1371/journal.pone.0173152

Rawat, S., and Meena, S. (2014). Publish or perish: where are we heading? J. Res. Med. Sci. 19, 87-89.

Schaufelbühl, A., Niya, S. R., Pelloni, L., Wullschleger, S., Bocek, T., Rajendran, L., et al. (2019). "EUREKA - a minimal operational prototype of a blockchainbased rating and publishing system," in 2019 IEEE International Conference on Blockchain and Cryptocurrency (Seoul).

Schimanski, L. A., and Alperin, J. P. (2018). The evaluation of scholarship in academic promotion in tenure processes: past, present, and future. F1000Res 7:1605. doi: 10.12688/f1000research.16493.1

ScienceMatters (2018). Publishing Process. Available online at: https://www. sciencematters.io/

Shamseer, L., Moher, D., Maduekwe, O., Turner, L., Barbour, V., Burch, R., et al. (2017). Potential predatory and legitimate biomedical journals: can you tell the difference? A cross-sectional comparison. BMC Med. 15:28. doi: 10.1186/s12916-017-0785-9

Skiba, D. J. (2017). The potential of blockchain in education and healthcare. Nurs. Educ. Perspect. 38, 220-221. doi: 10.1097/01.NEP.0000000000000190

Solomon, D. J., and Björk, B. (2011). Publication fees in open access publishing: sources of funding and factors influencing choice of journal. J. Assoc. Inf. Sci. Technol. 66, 98-107. doi: 10.1002/asi.21660

Treleaven, P., Gendal Brown, R., and Yang, D. (2017). Blockchain technology in finance. Computer 50, 14-17. doi: 10.1109/MC.2017.3571047 
Turkanović, M., Hölbl, M., Košič, K., Heričko, M., and Kamišalić, A. (2018). EduCTX: a blockchain-based higher education credit platform. IEEE Access 6, 5112-5127. doi: 10.1109/ACCESS.2018.2789929

United Nations Educational, Scientific and Cultural Organization (2017). Global Open Access Portal: Open Science Movement. Available online at: http://www.unesco.org/new/en/communication-and-information/portalsand-platforms/goap/open-science-movement/

Verde, F., Stanzione, A., Romeo, V., Cuocolo, R., Maurea, R., and Brunetti, A. (2019). Could blockchain technology empower patients, improve education, and boost research in radiology departments? An open question for future applications. J. Digit. Imaging. 32,1112-1115. doi: 10.1007/s10278-01900246-8

Welch, S. J. (2012). Selecting the right journal for your submission. J. Thorac. Dis. 4, 336-338. doi: 10.3978/j.issn.2072-1439.2012. 05.06

Young, S. N. (2009). Bias in the research literature and conflict of interest: an issue for publisher, editors, reviewers and authors, and it is not just about the money. J. Psychiatry Neurosci. 34, 412-417.

Zheng, Z., Xie, S., Dai, H., Chen, X., and Wang, H. (2017). "An overview of blockchain technology: architecture, consensus, and future trends," in Presented at the 2017 IEEE International Congress on Big Data (BigData Congress) (Honolulu, HI: IEEE).
Conflict of Interest: TM has received speaker fees and reimbursement for travel from Cardinal Health to present on blockchain and healthcare research. He is has also received reimbursement for travel expenses associated with speaking at IEEE-sponsored events and is a non-compensated advisory board member for the blockchain and pharmaceutical company FarmaTrust. He also is a Section Editor for Global Health at the journal BMC Public Health, published by SpringerNature. KM is the CEO of Ledgersafe Corporation, a blockchain startup company. KC is an Advisor for the blockchain company RemediChain and a Senior Health Technology Advisor for Solaster a company involved in distributed health ecosystems.

The remaining authors declare that the research was conducted in the absence of any commercial or financial relationships that could be construed as a potential conflict of interest.

Copyright $\odot 2019$ Mackey, Shah, Miyachi, Short and Clauson. This is an open-access article distributed under the terms of the Creative Commons Attribution License (CC $B Y)$. The use, distribution or reproduction in other forums is permitted, provided the original author(s) and the copyright owner(s) are credited and that the original publication in this journal is cited, in accordance with accepted academic practice. No use, distribution or reproduction is permitted which does not comply with these terms. 